Article

\title{
An Improved Anti-Interference Precoding of Large-Scale Fading System Based on Channel Inversion
}

\author{
Wei Lei ${ }^{+} \mathbb{C}$, Li Cheng *, Yu Lei, Zhengrong Li and Lianying Zou \\ School of Electrical and Information Engineering, Wuhan Institute of Technology, Wuhan 430000, China; \\ leiwei201403@wit.edu.cn (W.L.); leiyu@wit.edu.cn (Y.L.); 21903010012@wit.edu.cn (Z.L.); \\ zouly@wit.edu.cn (L.Z.) \\ * Correspondence: chengli8102@wit.edu.cn; Tel.: +86-1366-719-2520 \\ + Current address: School of Electrical and Information Engineering, Wuhan Institute of Technology, No.206, \\ Guanggu 1st Road, Donghu New High Technology Development Zone, Wuhan 430000, China.
}

Received: 23 December 2019; Accepted: 25 January 2020; Published: 27 January 2020

\begin{abstract}
Recently, a large-scale fading precoding (LSFP) for the wireless massive multiple-input, multiple-output (MIMO) systems has been proposed. In this precoding, the channel information of all the cells using re-use pilot sequences is processed jointly, and pilot contamination and interference due to a certain number of antennas are effectively eliminated. Additionally, recent studies have found that research in the asymptotic field can be applied to the wireless large-scale MIMO systems. In the LSFP, pilot contamination and signal interference will be completely eliminated when a number of antennas at a base station tend to be unlimited. In this research found that the LSFP method can eliminate most pilot contamination and interference in practical applications only when the number of antennas of the base station reaches hundreds of orders, which greatly increases the equipment construction cost. On the other hand, channel inversion denotes a multi-user channel modulation technology, where a vector signal generated between a user and a base station is used to form an inverse channel matrix so that the channels of each user are balanced during the transmission. In this paper, the channel inversion technology is used in the LSFP. The improved LSFP can effectively reduce the number of antennas required by the base station without affecting the performance of eliminating the pilot contamination and interference. It is shown that when the number of antennas of a base station tends to be unlimited, the improved LSFP can eliminate pilot contamination and signal interference. The simulation results show that in the same practical scenario, when the base station is equipped with the same number of antennas, the improved method can more effectively improve the anti-contamination and anti-interference performance over conventional LSFP.
\end{abstract}

Keywords: MIMO; precoding; contamination; interference

\section{Introduction}

A massive MIMO (multiple-input multiple-output) system entails a large number (tens or hundreds) of base station antennas serving a much smaller number of terminals. These systems demonstrate large gains in spectral and energy efficiency compared with the conventional MIMO technology [1]. Massive MIMO (multiple-input multiple-output) is no longer a "wild" or "promising" concept for future cellular networks. In 2018, the key ingredients of Massive MIMO have made it into the 5G standard. The development of fully digital Massive MIMO arrays for mmWave frequencies is well underway [2]. However, its research is prohibitively complicated and costly. In a few years, maybe Massive MIMO with fully digital transceivers will be a mainstream feature at both sub- $6 \mathrm{GHz}$ and mmWave frequencies. 
In [3], researchers focused on new communication paradigms arising in massive multiple-inputmultiple-output systems where the antenna array at the base station is of extremely large dimension. The main contributions of [3] are the design of low complexity scheme of multi-antenna transceiver and evaluate the bit-error-rate performance of the transceivers in crowded xMaMIMO scenarios. However, the size of the antenna array of the base station in this solution is extremely large, which will cause great equipment costs.

A massive MIMO system entails a large number (tens or hundreds) of base station antennas serving a much smaller number of terminals. As the number of antennas grows, the performance of a massive MIMO system gets limited by the interference caused by pilot contamination. Ashikhmin and Marzetta proposed (under the name of Pilot Contamination Precoding) large scale fading precoding (LSFP) and large scale fading decoding (LSFD) based on limited cooperation between base stations [1].

In [1], researchers focused on the performance gain of the uplink large scale fading decoding in the case of a finite number of base station antennas. It shows that the performance can be improved further by optimizing the transmission powers of the users and presents decentralized LSFD that uses limited cooperation between neighboring cells. However, the research is focus on the transmission performance of the uplink, and the performance optimization of the downlink is not fully considered.

As the number of antennas grows, however, the performance of a massive MIMO system gets limited by the interference, contamination and noise. The increasing demands for higher data-rate transmission in cellular networks bring the attentions to Large-scale antenna system (LSAS). LSAS demonstrates large gain in spectral-efficiency and energy-efficiency compared with conventional MIMO schemes [4].

In [5], a multi-cell cooperation method, called pilot contamination precoding (PCP), was proposed. It was found that in a single cell LSAS, when the number of antennas tends to infinity, the communication performance can be idealized, and noise and interference can be completely eliminated. However, when turning to multi-cell LSAS, the system performance is limited by directed inter-cell interference, resulted from unavoidable re-use of nonorthogonal pilot sequences in different cells. This effect is called pilot contamination. In [6], it was observed that if a channel has spatial correlation, then one can use this correlation to drastically reduce the pilot contamination interference in the asymptotic regime as $\mathrm{M} \rightarrow \infty$.

Until recently, it was believed that as the number of base station antennas tends to infinity, the performance of such systems is limited by directed inter-cellular interference caused by unavoidable re-use of training sequences (pilot contamination) by users in different cells. Large-scale fading precoding (LSFP) leads to the effective elimination of inter-cell interference. Crucially, the combining coefficients of LSFP depend only on the large-scale fading coefficients between the users and the base stations. These coefficients change slowly and their number does not depend on the number of base station antennas. Thus, the traffic between base stations stays constant even if the number of antennas tends to infinity. In [7], researchers derived a capacity lower bound for massive MIMO systems with LSFP and a finite number of base station antennas and considered optimal and suboptimal LSFP precodings that take into account all sources of interference. The simulations results showed that LSFP provides significant gain even for the case of moderate number of base station antennas [7].

However, the above anti-interference precoding has good performance only for a large number of antennas at a base station, reaching the value of hundreds to thousands, which increases the base station equipment costs and operating costs, and causes difficulties huge power consumption in practical applications. Namely, in order to improve the communication quality, hundreds of antennas need to be used, thus, the design complexity of the antenna array becomes too high.

On the other hand, channel inversion represents a multi-user channel modulation technology. The vector signal generated between a user and a base station is used to form an inverse channel matrix so that the channels of each user reach the equilibrium during the transmission [8]. In [9], researchers compared the MMSE (minimum mean squared error) channel inversion algorithm and BMSN (Block Maximum Signal-to-Noise ratio) algorithm, which commonly utilizes the channel 
regularization with the pseudo-noise in the MU-MIMO transmission. The performance evaluation can be carried out through computer simulation. In [10], researchers applied the regularized channel inversion (RCI) combined with one of the $5 \mathrm{G}$ waveform candidates, namely generalized frequency division multiplexing (GFDM), in multiuser MIMO downlink systems and presents how the RCI in the multiuser MIMO-GFDM systems performs such good results as the RCI implemented in the multiuser MIMO-OFDM (Orthogonal Frequency Division Multiplexing) systems based on bit error rate (BER) parameter without causing a significant increase in system complexity.

The design idea of the precoding LSFP based on the zero-forcing algorithm is relatively simple, but it can only effectively eliminate interference such as pilot pollution when the number of base station antennas is very large. Optimizing the design of the precoding LSFP can ensure that interference is effectively eliminated. However, it needs to increase the power of the transmitting device to achieve efficient communication.

Aiming at the problem that the number of base station antennas of existing LSFP precoding is too large, the construction cost is too high. Based on the zero-forcing precoding algorithm and the optimized precoding algorithm, this paper applies channel inversion to improve and optimize the precoding. In the case of fewer base station antennas, interference such as pilot pollution and noise is suppressed, and the channel transmission rate is not affected at the same time, thereby achieving the purpose of further increasing the system rate and increasing the SINR.

In this work, channel inversion technology is used to improve the standard large scale fading precoding (LSFP), obtaining an improved anti-interference precoding, which can effectively eliminate pilot contamination, noise and interference using a small number of antennas. First, we applied the channel inversion technique to derive the theoretical formula for the zero-forcing large scale fading precoding algorithm to obtain its SINR expression. Under ideal circumstances, we analyzed and proved that the improved zero-forcing precoding can achieve the ideal anti-interference performance. The communication transmission rate performance was verified in the simulation. Secondly, the channel inversion technique was used to derive the theoretical formula of the optimized precoding algorithm. The SINR expression was obtained. The performance of the communication transmission rate was verified in the simulation. Finally, the regularized channel inversion technique was used to derive the theoretical formula for the optimized precoding algorithm. Its SINR expression was obtained. The performance of the communication transmission rate was verified in simulation. The SINR simulation expression of LSAS precoding of massive MIMO system was verified by simulation. The simulation compares the precoding communication rate performance before and after improvement. The simulation results show that reducing the number of antennas has no effect on the communication transmission rate. After applying the improved method to downlink transmission precoding, the communication transmission rate can be effectively improved.

The remaining of this article is organized as follows. Section 2 describes the system model used in this paper, including the channel model and orthogonal frequency division multiplexing scheme. Section 3 employs the channel inversion to improve the LSFP zero-forcing precoding, optimizes the precoding algorithm and performs the theoretical analysis based on the improved anti-interference precoding matrix, and lastly, obtains the SINR and channel transmission rate. Section 4 sets the infinite number of antennas in the asymptotic field, and performs the progressive analysis of the improved anti-interference precoding to determine the channel anti-interference transcoding technology combined with the anti-jamming precoding that can produce benefits. Section 5 presents the simulations of the channel transmission rate in the limited antenna field and compares the performance of the improved LSFP precoding and conventional LSFP precoding. Section 6 concludes the paper.

In this article, ZF-LSFP, A-ZF-LSFP, O-LSFP, AO-LSFP, MMSE-AO-LSFP represent zero-forcing precoding, advanced zero-forcing precoding, optimal LSFP precoding, advanced optimal LSFP precoding, MMSE pre-equalization advanced optimal LSFP precoding, respectively.

Throughout the paper, we use $A^{T}, A^{*}, \operatorname{Tr}[A]$ and $A_{(m, n)}$ for matrix transposition, Hermitian transposition, the trace of matrix and the $(\mathrm{m}, \mathrm{n})$-th entry of matrix $\mathrm{A}$, respectively. 


\section{System Model}

In this paper, the LSAS is set as an OFDM based cellular mobile communication network. In this kind of cellular network, the cells within a group use different OFDM orthogonal sub-channels, and the number of cells in the group is set to L [7]. The cell represents a regular hexagonal cell network. Each cell is equipped with a multi-antenna base station, and the base station can provide services to multiple users in the cell. The number of antennas of the base station is set to $M$, and each antenna is an omnidirectional antenna. The number of users in the cell is set to $\mathrm{K}$, and each user terminal is equipped with an omnidirectional antenna. Accordingly, a cell constitutes a multi-user MIMO system, where K independent users can form a virtual antenna array with number of antennas $\mathrm{K}$. The configuration of the base station and the user end constitutes a MIMO downlink system with the size of KM, or a MIMO uplink system with the size of MK. In the traditional single-user MIMO systems, the total rate capacity of the uplink and downlink channels of a multi-user MIMO system is proportional to $\min (\mathrm{M}, \mathrm{K})$.

In the OFDM-based cellular communication network, due to frequency reuse, the channel spectrum utilization is very high and the system communication performance is greatly improved [11]. We further assume that Orthogonal Frequency-Division Multiplexing (OFDM) is used and that in a given subcarrier we have a flat-fading channel [7]. In order to simplify the presentation of the multi-user MIMO system and reduce complex settings, we set that all the subcarriers in the communication frequency band can be used for communication between base stations and users.

\subsection{Channel Model Settings}

In a cellular network OFDM-based system, one block group allocates all orthogonal subcarrier channels, and the other block group reuses subcarrier channels. For a cell in a cellular network, which uses an OFDM subcarrier channel, the channel factor is expressed as a correlation operation between a large-scale attenuation factor and a small-scale attenuation factor, which is expressed as in Figure 1.

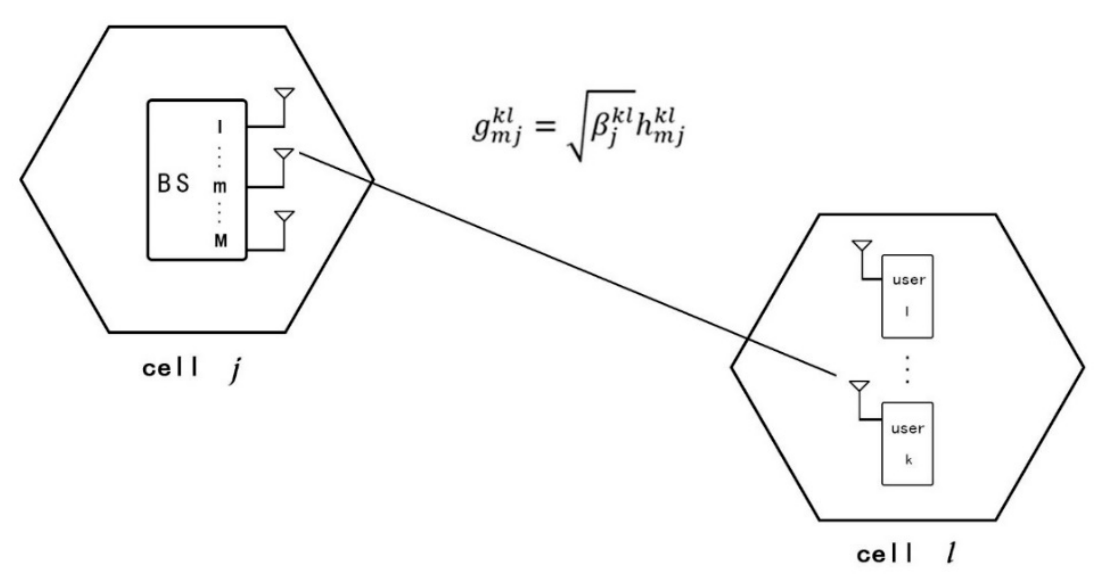

Figure 1. Cell $j$ antenna and cell $l$ user channel factors.

Cell $j$ indicates the $j$-th cell in the area group, and the number of antennas of a cell base station is $\mathrm{M}$; cell $l$ indicates the $l$-th cell in the area group, and the number of cell users is $K$. The channel factor between the $m$-th antenna of a base station in the $j$-th cell and the $k$-th user in the $l$-th cell can be expressed as:

$$
g_{m j}^{k l}=\sqrt{\beta_{j}^{k l}} h_{m j}^{k l}
$$

where $h_{m j}^{k l}$ denotes a fast-fading factor which is a small-scale fading factor. It is mainly caused by a multipath propagation and modeled as i.i.d. $C N(0,1)$ random variables. 
Furthermore, $\beta_{j}^{k l}$ denotes a slow fading factor which is a large-scale fading factor, mainly caused by the building blocking. We model the large-scale fading coefficient $\beta_{j}^{k l}$ as a log-normal distribution based on the Urban Macro model. The specific value of $\beta_{j}^{k l}$ is a positive real number, which is determined by shadowing and distance between the corresponding user and base station and it is given by:

$$
10 \log _{10} \beta_{j}^{k l}=-139.5-35 * d_{j}^{k l}+\psi
$$

where $\psi$ denotes the shadow fading coefficient with i.i.d. $N\left(0, \sigma_{\psi}^{2}\right)$ distribution and $d_{j}^{k l}$ denotes the distance, measured in kilometer, between the $j$-th BS and the $k$-th user in the $l$-th cell.

\subsection{OFDM Duplex Setting}

In this article, a multi-user MIMO system that used the OFDMA (Orthogonal Frequency Division Multiple Access) technology is studied. In the OFDMA, each user is assigned to a group of subcarriers, and the number of subcarriers for each user in each cell is defined by the system scheduling. For the sake of simplicity, it is assumed that the system model uniformly sets $\mathrm{K}$ users in each cell, that is, $\mathrm{K}$ subcarriers. The communication link between a base station and a user is divided into downlink and uplink duplex modes. We consider LSAS (large scale antenna system) with the TDD (Time Division Duplexing) protocol. The TDD protocol was set up with three steps: Tu channel was used for uplink training; Tpchannel was used for processing precoding; Td channel was used for downlink training.

In TDD, the uplink and downlink transmissions were allocated to different communication time slots and could not be performed simultaneously; thus, the transmission and reception of a user signal was completed through the assigned subcarriers. However, they were performed at different times, and the corresponding time slots could be dynamically and flexibly allocated. Due to the reciprocity of uplink transmission and downlink transmission, the MIMO technology and antenna technology could be combined with time division duplexing.

\section{Advanced LSFP}

\section{1. $L S F P$}

In the LSFP (large scale fading precoding) network, several assumptions were made, and they are as follows.

(1) Any base station in a cell can accurately estimate and obtain a large-scale fading factor between itself and any user in the group.

(2) Any base station in a cell can send a large-scale fading factor matrix to any user in the cell.

(3) All base stations in a cellular network are connected to the same network hub controller. The large-scale fading factor of the entire cellular network is visible to this network hub controller.

(4) Any downlink signal in a cellular network is visible to this network hub controller.

By adjusting the hardware of the base station equipment and using the reciprocity of uplink and downlink [12,13], the network hub controller in a cluster generates a LSFP matrix denoted as $W^{k}$ based on the large-scale fading factor $\beta_{j}^{k l}[14]$ :

$$
W^{k}=\left(\begin{array}{c}
W_{1}^{k} \\
\vdots \\
W_{L}^{k}
\end{array}\right), k \in(1, K)
$$

The was is specifically divided into the precoding of downlink information from a base station to the user, and the decoding of uplink information from a user to the base station. 


\subsubsection{Downlink LSFP}

In Step 1, base station of a cell performs channel estimation according to the uplink signal and uplink pilot training sequence sent by a user to obtain a large-scale attenuation factor and sends it to the network hub controller of the cellular network.

In Step 2, the network hub controller receives the large-scale fading factors sent by all the base stations in the area group and forms a large-scale fading factor precoding matrix.

In Step 3, the network hub controller sends the large-scale fading factor precoding matrix to each base station.

In Step 4, before the base station sends the information to a user, it performs a precoding operation on the signal according to the large-scale fading factor precoding matrix.

In the Downlink PCP (pilot contamination precoding) proposed for the downlink pilot contamination precoding [5], the PCP matrix is expressed as:

$$
W^{k}=\left(B^{k}\right)^{-1}
$$

where $B^{k}$ denotes a matrix composed of a large-scale fading factor $\beta_{j}^{k l}$, that is, the zero-forcing pilot contamination precoding (ZF-PCP).

In the LSFP proposed in [8], the LSFP matrix is expressed as:

$$
W^{k}=\gamma_{A}\left(B^{k}\right)^{-1}
$$

where $\gamma_{A}$ denotes a normalization factor that defines the power limit and $B^{k}$ denotes a matrix composed of the large-scale fading factor. $\beta_{j}^{k l}$, that is, the zero-forcing large-scale fading precoding (ZF-LSFP).

By applying the asymptotic theory, it has been proven that systems using zero-forcing precoding can achieve communication performance. At a limited number of antennas, the ZF-PCP and ZF-LSFP with zero-forcing precoding achieve average performance, and there are also pilot contamination, noise and interference in the LSAS. In [4,7], an optimized precoding scheme named the Optimized LSFP (O-LSFP) was proposed; this scheme aims to optimize the precoding matrix in the field of limited antennas.

\section{Lemma 1.}

$$
\begin{gathered}
W=\left[\begin{array}{lll}
\left(W^{1}\right)^{T} & \cdots & \left(W^{K}\right)^{T}
\end{array}\right]^{T} \\
B=\operatorname{Diag}\left[\begin{array}{lll}
B^{1} & \cdots & B^{K}
\end{array}\right] \\
\rho_{j}^{k l n}=\left[\begin{array}{lll}
\frac{\delta_{u}^{2}}{\varphi_{f} L}+\beta_{j}^{k l}
\end{array}\right]\left[\begin{array}{lll}
\frac{\delta_{d}^{2}}{\varphi_{r}}+T & \sum_{s} \beta_{j}^{n s}
\end{array}\right] \\
P^{k l n}=\operatorname{Diag}\left[\begin{array}{lll}
\rho_{1}^{k l n} & \cdots & \rho_{L}^{k l n}
\end{array}\right] \\
P^{k l}=\operatorname{Diag}\left[\begin{array}{lll}
P^{k l 1} & \ldots & P^{k l K}
\end{array}\right]
\end{gathered}
$$

$W$ denotes an O-LSFP matrix, B denotes a large-scale fading factor matrix and $P^{k l}$ denotes a matrix obtained by a large-scale fading factor $\beta_{j}^{k l}$ operation.

The SINR expression, after applying the O-LSFP matrix is as follows:

\section{Lemma 2.}

$$
\operatorname{SINR} R^{k l}=\frac{T_{3}^{k l}}{T_{1}^{k l}+T_{2}^{k l}}
$$




$$
\begin{gathered}
T_{1}^{k l}=\frac{\operatorname{Tr}\left[(W)^{*} P^{k l} W\right]}{M T} \\
T_{2}^{k l}=\sum_{v \neq l}\left|B W_{(k L+L-l), v}\right|^{2} \\
T_{3}^{k l}=\left|B W_{(k L+L-l), l}\right|^{2}
\end{gathered}
$$

The simulation results presented in [4,7] show that the number of antennas at a base station must be at the hundred-level to eliminate most of the pilot contamination, noise and interference. However, such a configuration of antennas will inevitably increase the cost of the base station equipment. In [7], the simulation assumed that each base station is equipped with $\mathrm{M}$ omnidirectional antennas and each user has a single omnidirectional antenna. In practice, there will be unnecessary power consumption, failing to utilize antenna resources fully and increasing the operating costs. Therefore, in this paper, the LSFP is improved to reduce the pilot contamination, noise and interference performance without reducing the number of antennas at a base station. At the same time, if the number of antennas at a base station antennas stays unchanged, the improved LSFP precoding scheme can improve the performance. Communication transmission rate to improve LSAS communication performance. It means that the improved LSFP precoding scheme can more effectively improve the anti-contamination and anti-interference performance over conventional LSFP as the number of antennas at a base station antennas stays unchanged.

\subsubsection{Uplink LSFP}

Similarly, the uplink transmission process consists of the following steps.

In Step 1, network hub controller of a group receives the large-scale fading factors sent by all base stations in the group and forms a large-scale fading factor decoding matrix.

In Step 2, network hub controller sends the large-scale fading factor decoding matrix to each base station.

In Step 3, when a base station receives the information sent by the user, the signal is decoded according to the large-scale fading factor decoding matrix.

\subsection{Advanced LSFP}

In this paper, channel inversion technology was applied to the ZF-LSFP and O-LSFP to obtain an improved anti-interference precoding named the Advanced LSFP (A-LSFP). Channel inversion belongs to precoding technology. In the downlink communication, channel inversion can be used to eliminate data interference at a base station. In this work, channel inversion and regularized channel inversion techniques are implemented in the multi-cell LSAS. Channel inversion is similar to the zero-forcing pre-equalization technique in the single-user MIMO systems, while regularized channel inversion is similar to the MMSE pre-equalization technique in the single-user MIMO systems.

In the pre-equalization technology, weighting matrix $W$ represents the pre-equalizing weighting matrix. When the zero-forcing pre-equalization is used, the corresponding weighting matrix $W$ is expressed as:

$$
\begin{gathered}
W=\beta / H_{D}^{D L} \\
\beta=\sqrt{\frac{M}{\operatorname{Tr}\left[\left(H_{D}^{D L}\right)^{-1} \times\left(H_{D}^{D L}\right)^{H}\right]}}
\end{gathered}
$$

where $\beta$ denotes a constant that keeps the total power constant after pre-equalization. 
By applying the channel inversion technology to the LSAS and substituting $\beta$ into the LSFP matrix, the Advanced ZF-LSFP (A-ZF-LSFP) is obtained, which can be expressed as follows:

$$
\begin{gathered}
W^{k}=\gamma_{A} * \beta^{\prime} *\left(B^{k}\right)^{-1} \\
\beta^{\prime}=\sqrt{\frac{M}{\operatorname{Tr}\left[\left(B^{k}\right)^{-1} \times\left(B^{k}\right)^{H}\right]}}
\end{gathered}
$$

where $W^{k}$ denotes a large-scale fading factor matrix for channel inversion processing and $\gamma_{A}$ denotes a normalization factor that guarantees power limitation. $\beta^{\prime}$ represents a constant that keeps the total power constant after pre-equalization.

Substitute the ZF-LSFP (A-ZF-LSFP) to which channel inversion technology is applied:

$$
\operatorname{SINR} R^{k l}=\frac{T_{3}^{k l}}{T_{1}^{k l}+T_{2}^{k l}}
$$

The SINR of the A-ZF-LSFP can be expressed as:

$$
\operatorname{SINR}^{k l}=\frac{1}{T_{1}^{k l}}=\frac{M T}{\operatorname{Tr}\left[(W)^{*} P^{k l} W\right]}
$$

In the LSAS, the channel inversion technology is applied to optimize precoding. The Optimized LSFP (O-LSFP), and the Advanced O-LSFP (AO-LSFP) can be obtained:

$$
W^{\prime}=\left[\begin{array}{lll}
\left(W^{1}\right)^{T} & \cdots & \left(W^{K}\right)^{T}
\end{array}\right]^{T}=\left[\begin{array}{lll}
\left(\gamma_{A} * \beta *\left(B^{1}\right)^{-1}\right)^{T} & \cdots & \left(\gamma_{A} * \beta *\left(B^{K}\right)^{-1}\right)^{T}
\end{array}\right]^{T}
$$

$W^{\prime}$ denotes the A-O-LSFP precoding matrix for channel inversion processing.

Applying $W^{\prime}$ to Lemmas 1 and 2, it is easy to get the SINR expression of A-O-LSFP. The SINR of the A-O-LSFP can be expressed as:

$$
\begin{gathered}
\operatorname{SINR}^{k l}=\frac{T_{3}^{k l}}{T_{1}^{k l}+T_{2}^{k l}} \\
T_{1}^{k l}=\frac{\operatorname{Tr}\left[\left(W^{\prime}\right)^{*} \beta^{k l} W^{\prime}\right]}{M T} \\
T_{2}^{k l}=\sum_{v \neq l}\left|B W^{\prime}{ }_{(k L+L-l), v}\right|^{2} \\
T_{3}^{k l}=\left|B W^{\prime}{ }_{(k L+L-l), l}\right|^{2} \\
B=\operatorname{Diag}\left[\begin{array}{lll}
B^{1} & \ldots & B^{K}
\end{array}\right] \\
\rho_{j}^{k l n}=\left[\begin{array}{lll}
\frac{\delta_{u}^{2}}{\varphi_{f} L}+\beta_{j}^{k l}
\end{array}\right]\left[\begin{array}{ll}
\frac{\delta_{d}^{2}}{\varphi_{r}}+T & \sum_{s} \beta_{j}^{n s}
\end{array}\right] \\
P^{k l n}=\operatorname{Diag}\left[\begin{array}{lll}
\rho_{1}^{k l n} & \ldots & \rho_{L}^{k l n}
\end{array}\right] \\
P^{k l}=\operatorname{Diag}\left[\begin{array}{lll}
P^{k l 1} & \ldots & P^{k l K}
\end{array}\right]
\end{gathered}
$$

where $B$ represents a large-scale fading factor matrix and $P^{k l}$ represents a matrix obtained by using a large-scale fading factor $\beta_{j}^{k l}$ operation. 
In the MMSE pre-equalization, the corresponding weighting matrix $W$ can be expressed as:

$$
\begin{gathered}
W=\beta^{\prime} \times\left(H_{D}^{D L}\right)^{H}\left[H_{D}^{D L} \times\left(H_{D}^{D L}\right)^{H}+\frac{\delta_{z}^{2}}{\delta_{x}^{2}} \times E\right]^{-1} \\
\beta^{\prime}=\sqrt{\frac{M}{\operatorname{Tr}\left[\left(H_{D}^{D L}\right)^{-1} \times\left(H_{D}^{D L}\right)^{H}\right]}}
\end{gathered}
$$

where $\beta^{\prime}$ denotes a constant that keeps the total power constant after pre-equalization.

By applying regular channel inversion technology to the LSAS, substituting $\beta^{\prime}$ into the LSFP matrix, and using the MMSE pre-equalization weighting matrix $W$ to represent the O-LSFP matrix, the MMSE Advanced O-LSFP (MMSE-AO-LSFP) can be obtained as follows:

$$
\begin{gathered}
W^{k}=\gamma_{A} \times \beta^{\prime} \times\left(B^{k}\right)^{H}\left[B^{k} \times\left(B^{k}\right)^{H}+\frac{\delta_{z}^{2}}{\delta_{x}^{2}} \times E\right]^{-1} \\
\beta^{\prime}=\sqrt{\frac{M}{\operatorname{Tr}\left[\left(B^{k}\right)^{-1} \times\left(B^{k}\right)^{H}\right]}} \\
W^{\prime \prime}=\left[\begin{array}{lll}
\left(W^{1}\right)^{T} & \cdots & \left(W^{K}\right)^{T}
\end{array}\right]^{T}
\end{gathered}
$$

where $W^{k}$ denotes a matrix obtained by performing regular channel inversion processing on a large-scale fading factor matrix.

$W^{\prime \prime}$ denotes the MMSE-A-O-LSFP precoding matrix for regularized channel inversion processing.

Lemma 3. In this work, the theorem presented in [10] was used, and it is as follows:

$$
R_{D}^{k l} \geq \log _{2}\left(1+\operatorname{SINR} R_{D}^{k l}\right)
$$

The large-scale fading factor $\beta_{j}^{k l}$ can be applied to obtain the data transmission rate, which is expressed as follows:

$$
R^{k l}=\log _{2}\left(1+S I N R^{k l}\right)
$$

At the receiving end, in order to compensate for the amplification effect of a transmitter, the receiver uses the automatic gain control technology to adjust the received signal so that the received signal is multiplied by $\frac{1}{\beta}$, and then the received signal of the same strength is restored.

\section{Performance Analysis}

When the number of antennas of a base station approaches infinity, the pre-coded A-ZF-LSFP and AO-LSFP can achieve excellent communication performance after channel inversion, and in the same network group, all users will reach the theoretically maximal network transmission rate.

First, before the asymptotic analysis, the most suitable LSFP matrix is determined. According to the previous analysis, the channel inversion technology is applied to LSFP to obtain a suitable precoding matrix. The zero-forcing A-ZF-LSFP matrix for channel inversion processing is obtained as:

$$
W^{k}=\gamma_{A} * \beta *\left(B^{k}\right)^{-1}=\varphi_{A} *\left(B^{k}\right)^{-1}
$$

where $\gamma_{A}$ denotes the normalization factor that guaranteed the power limit and $\varphi_{A}=\gamma_{A} * \beta$ denotes the normalization factor for the zero-forcing precoding A-ZF-LSFP. 


\section{Lemma 4.}

$$
\begin{gathered}
R_{D}^{k l} \geq \log _{2}\left(1+\operatorname{SIN} R_{D}^{k l}\right) \\
\operatorname{SIN} R_{D}^{k l}=\frac{J_{0}^{k l}}{J_{1}^{k l}+J_{2}^{k l}+\frac{1}{M}} \\
J_{0}^{k l}=M \rho_{f} \rho_{r} \tau \mid \sum_{j=1}^{L} \beta_{j}^{k l} \alpha_{j}^{k l}{ }^{2} \\
J_{1}^{k l}=\rho_{f} \rho_{r} \tau \sum_{v=1, v \neq l}^{L}\left|\sum_{j=1}^{L} \beta_{j}^{k l} \alpha_{j}^{k l}\right|^{2} \\
J_{2}^{k l}=\rho_{f} \sum_{j=1}^{L}\left[\sum_{n=1}^{K} \beta_{j}^{k l}\left(1+\sum_{s=1}^{L} \rho_{r} \tau \beta_{j}^{n s}\right)\right]\left[\sum_{v=1}^{L}\left|\alpha_{j}^{k n v}\right|^{2}\right]
\end{gathered}
$$

Among them:

$$
\alpha_{j}^{k l}=\frac{\sqrt{\rho_{r} \tau} \beta_{j}^{k l} \omega_{j}^{k l}}{1+\sum_{s=1}^{L} \rho_{r} \tau \beta_{j}^{k s}}, \beta_{j}^{k l} \in B^{k}, \omega_{j}^{k l} \in W^{k}
$$

Therefore, when $M \rightarrow \infty, J_{1}^{k l}=0, J_{1}^{k l}=M \rho_{f} \rho_{r} \tau$ we could get the idealized SINR of the A-ZF-LSFP:

$$
\lim _{M \rightarrow \infty} \operatorname{SINR}_{D}^{k l}=\frac{M \rho_{f} \rho_{r} \tau}{J_{2}^{k l}+\frac{1}{M}}=\infty
$$

In the OFDM communication system, it means that noise and interference can be removed when the number of antennas tends to be infinite.

In practical applications, the number of antennas in the LSAS is often tens or hundreds. In the following section, the channel transmission rate of the optimized precoding before and after channel inversion is compared in detail to prove that the optimized LSFP after channel inversion processing can achieve the same anti-contamination and anti-interference performance as equipped with a smaller number of base antennas than conventional LSFP.

\section{Simulation}

The performance of anti-interference precoding was analyzed in terms of the communication transmission rate. The simulation graph represents the cumulative distribution function (CDF), which can reflect the received signal-to-interference and noise ratio and the information transmission throughput rate [15]. In the simulation, the performance of communication transmission rate of improved and conventional precoding schemes, which includes ZF-LSFP, AZF-LSFP, O-LSFP, AO-LSFP and MMSE-AO-LSFP, is compared under different base antenna numbers.

The simulation model was based on Section 2 channel model setting. We model the large-scale fading coefficient $\beta_{j}^{k l}$ as log-normal distribution based on the Urban Macro model that followed the 3GPP (3rd Generation Partnership Project) standard. Cell base stations and user settings in Figure 2: the number of cells contained in a single cell group was $L=19$, the number of users contained in a single cell was $K=10$, and the number of cell base station antennas was $M$. The cell was randomly distributed to ten mobile users. The cell radius was $r=1 \mathrm{~km}$. The cell base station was set in the center. The mobile user was randomly located in a circular area having the base station as a center, a radius of $62.5 \mathrm{~m}$. 


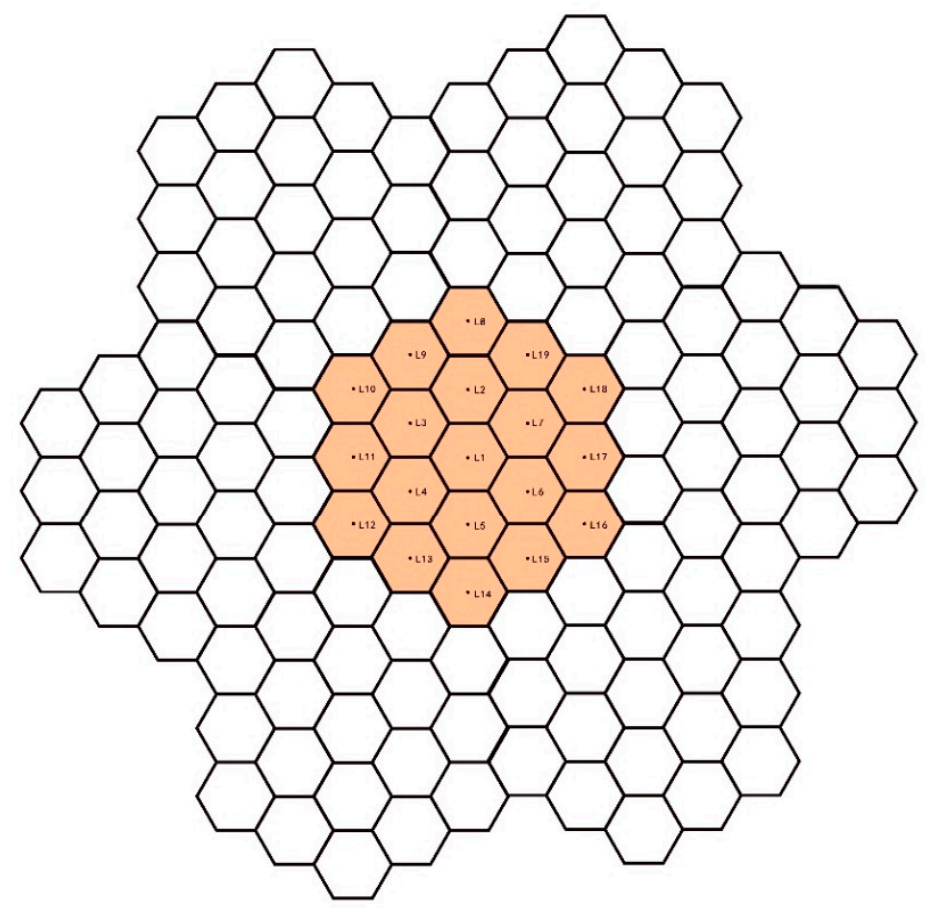

$$
L=19
$$

Figure 2. A cellular network composed of 19 hexagonal cells.

The cell numbers in the area group were set to $L_{1}, 1=1, \cdots, 19$. Each cell center point was set with two-dimensional coordinates and the $\mathrm{L}_{1}$ coordinate is $\mathrm{L}_{1}(0,0)$. According to the cell radius, the coordinates of all cell center points $\mathrm{L}_{1}\left(\mathrm{~m}_{1}, \mathrm{n}_{1}\right)$ can be generated.

The distribution map of users of a single cell is presented in Figure 3 left, where each dot represents a user. The user distribution range was set in the simulation, and the generated points were randomly distributed. Randomly selecting a hexagonal cell, and the center point is the base station position. The positions of 10 users in this cell are randomly generated. The user positions are evenly distributed in the cell. We eliminate the extreme case that all users are distributed at the cell edge.
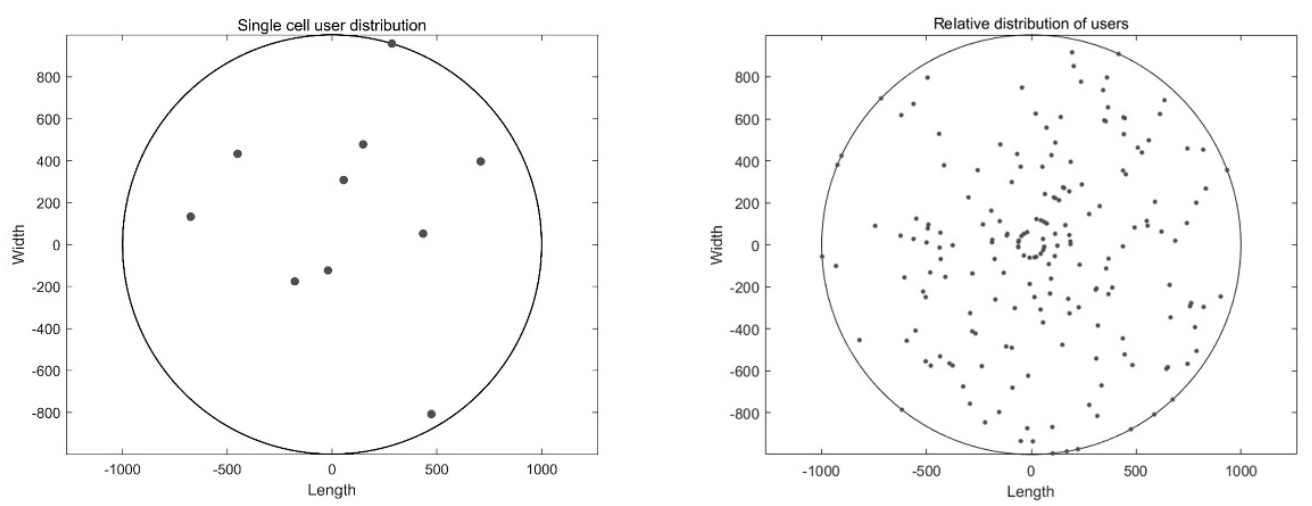

Figure 3. Distribution of users in a single cell and Relative distribution of all users in the area.

A random distribution diagram of a total of 190 users grouped into 19 cells within the cellular network to which they belonged is presented in Figure 3 right. The relative distance between a user and 19 base stations was obtained using the same principle as in spatial channel model for multiple input multiple output (MIMO) simulations of Technical specification group radio access network. 
There are 19 communities in the cellular network, with a total of $19 \times 10$ users. The location of 190 users is randomly generated in a hexagonal cell with the center point coordinate of $(x, y)$, and a group of 10 users Substitute the coordinates of 19 center points $\mathrm{L}_{1}\left(\mathrm{~m}_{1}, \mathrm{n}_{1}\right), 1=1, \cdots, 19$ in turn, and obtain the coordinate of all users in each cell. Then, we can calculate the distance $d_{j}^{k l}$ according to coordinates of the $j$-th BS and the $k$-th user in the $l$-th cell.

Considering the movement of end users, it was assumed that users move freely in the cell to which they belong, but irrespective of the extreme case where all users move to the edge of the cell. The distribution of users in the cell is still roughly evenly distributed. The positions of 190 users were randomly generated in the hexagonal cell with the center point coordinate of $(x, y)$ for many times. The user position distribution changes as shown in the point in Figure 4, which simulates the free movement of users in each cell.
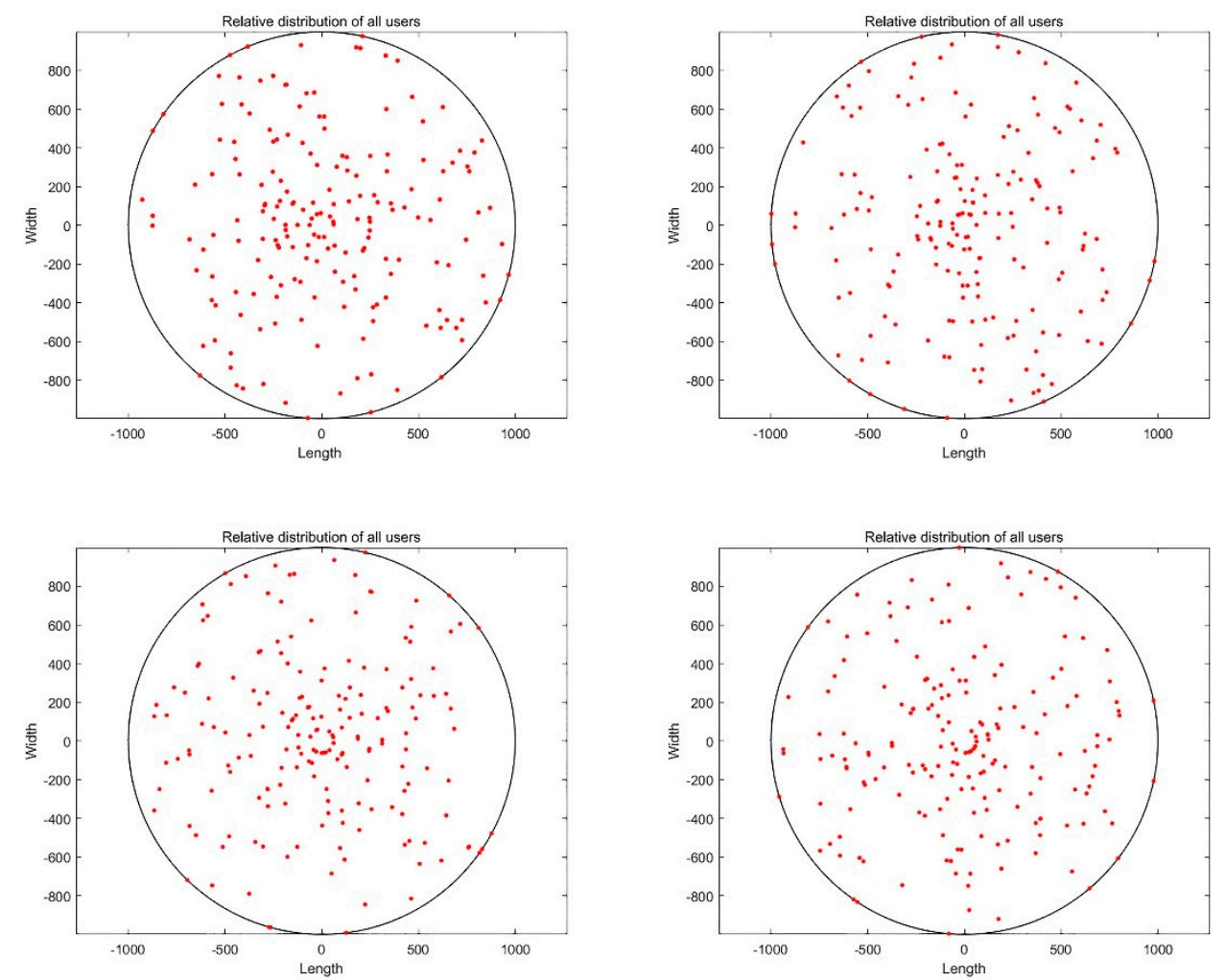

Figure 4. Relative distribution of all users in the area.

According to the set system model, a large-scale fading factor $\beta_{j}^{\mathrm{kl}}$ was obtained using the 3GPP urban macro model, which is given by (2). Other simulation settings: the transmit powers of the BS and users were $\rho_{r}=23 \mathrm{dBm}$ and $\rho_{\mathrm{f}}=23 \mathrm{dBm}$, respectively. The noise figures of the $\mathrm{BS}$ and user were 5 and 10, respectively; the bandwidth was $20 \mathrm{MHZ}$.

In the simulation, different numbers of base station antennas were used, $M$ was set to 100,1000 , 10,000 , and 1,000,000. Channel transmission rate can be calculated by (36). The simulation results show the lowest achievable rate in a cellular network group.

In Figure 5, the simulation results are compared with those of the ZF-LSFP that are provided in [4]. The comparison results show that when $\mathrm{M}$ reached the value of $10^{6}$, the achievable channel transmission rate of the A-ZF-LSFP reached the value of $10^{\circ}$, and the performance of channel transmission rate increased by $60 \%$. When $\mathrm{M}$ was between $10^{2}$ and $10^{4}$, the channel transmission rate of the A-ZF-LSFP increased by $90 \%$. 


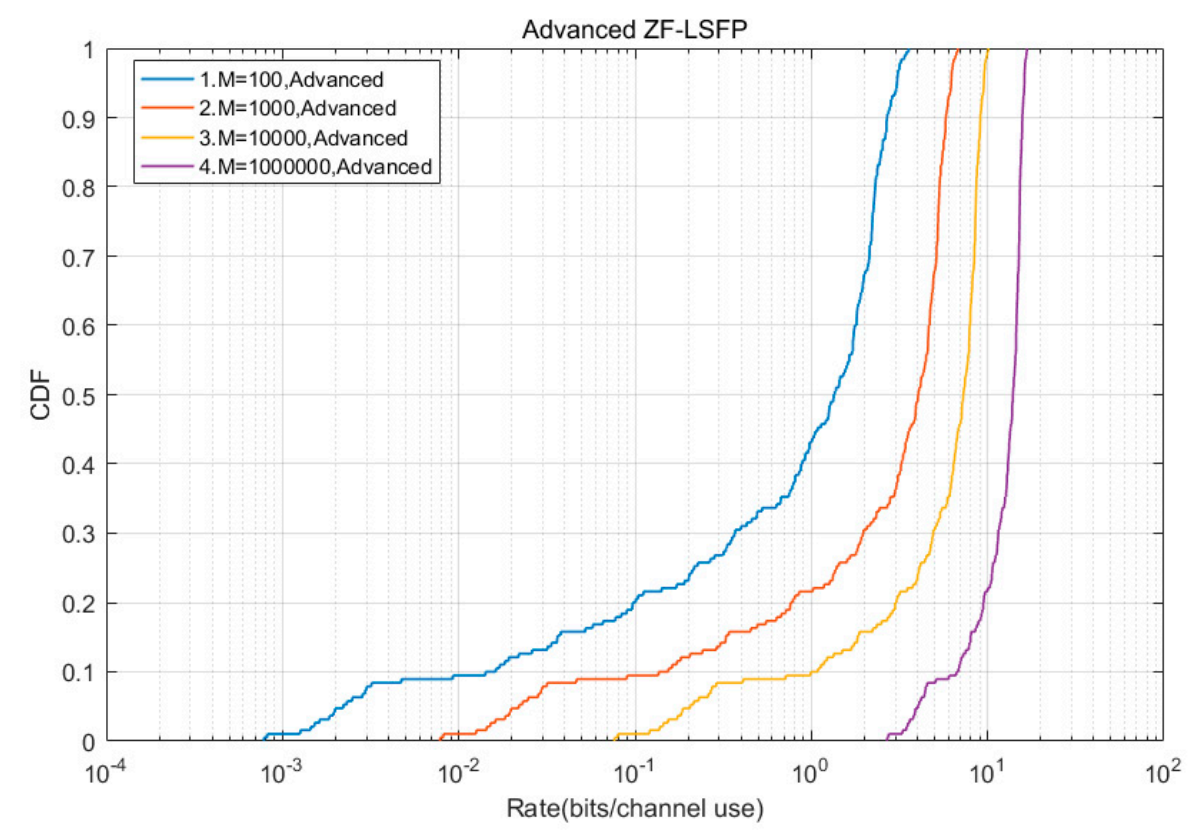

Figure 5. CDF of A-ZF-LSFP channel can achieve transmission rate.

As Figure 6 shows, when the number of antennas was 100, the probability that the unimproved optimized precoding could achieve a channel transmission rate greater than $10^{0}$ was about 0.8 , and the probability of reaching the value of $10^{1}$ was zero; when the number of antennas was 1000 , the probability that the O-LSFP could achieve a channel transmission rate greater than $10^{0}$ was about 0.9 , and the probability of reaching the value of $10^{1}$ was close to zero.

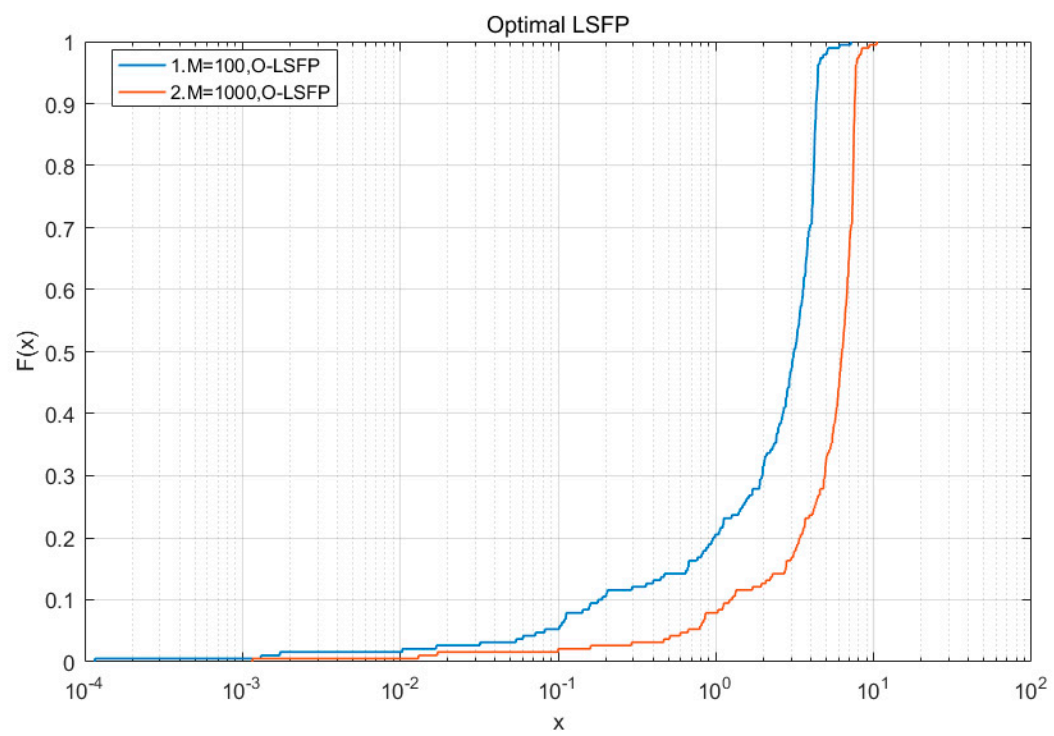

Figure 6. CDF of O-LSFP can achieve transmission rate.

As Figure 7 shows, when the number of antennas was 100, the probability that the optimized precoding with channel inversion processing could achieve a channel transmission rate greater than $10^{0}$ was about 0.9 , and the probability of reaching the value of $10^{1}$ was 0.05 ; when the number of antennas was 1000, the probability that the A-O-LSFP could achieve a channel transmission rate greater than $10^{0}$ was about 0.96 , and the probability of reaching the value of $10^{1}$ was about 0.5 . In the simulations, when the number of antennas was a few hundreds, the transmission rate of the A-O-LSFP was increased by at least $10 \%$. 


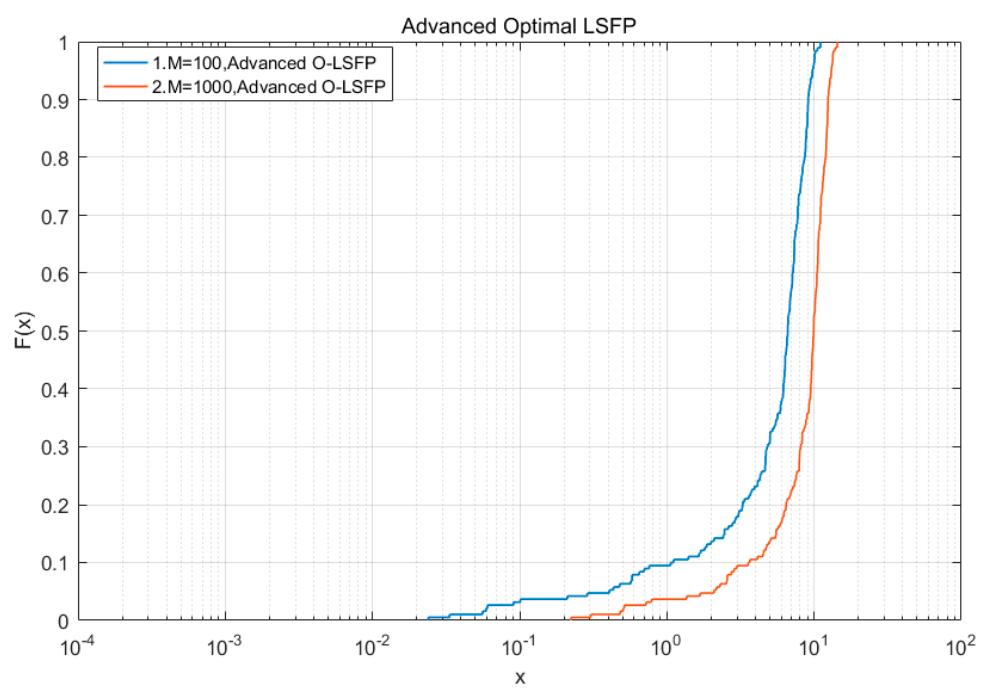

Figure 7. CDF of A-O-LSFP channel can achieve.

Figure 8 shows that when the number of antennas was 100, the probability that the optimized precoding with regular channel inversion processing could achieve a channel transmission rate greater than $10^{0}$ was about 0.7 , and the probability that it could reach the value of $10^{1}$ was 0.15 ; when the number of antennas was 1000, the probability that the MMSE-A-O-LSFP could achieve a channel transmission rate greater than $10^{0}$ was about 0.9 , and the probability of reaching the value of $10^{1}$ was about 0.25 . The simulation results showed that when the number of antennas was about 100, the MMSE-A-O-LSFP had a probability of $15 \%$ to reach 10 times the transmission rate gain, but there was still a $10 \%$ attenuation probability. The MMSE-A-O-LSFP had a probability of reaching 10 times the transmission rate gain of $25 \%$, and there was no attenuation of the transmission rate gain, and the improved performance is completely improved.

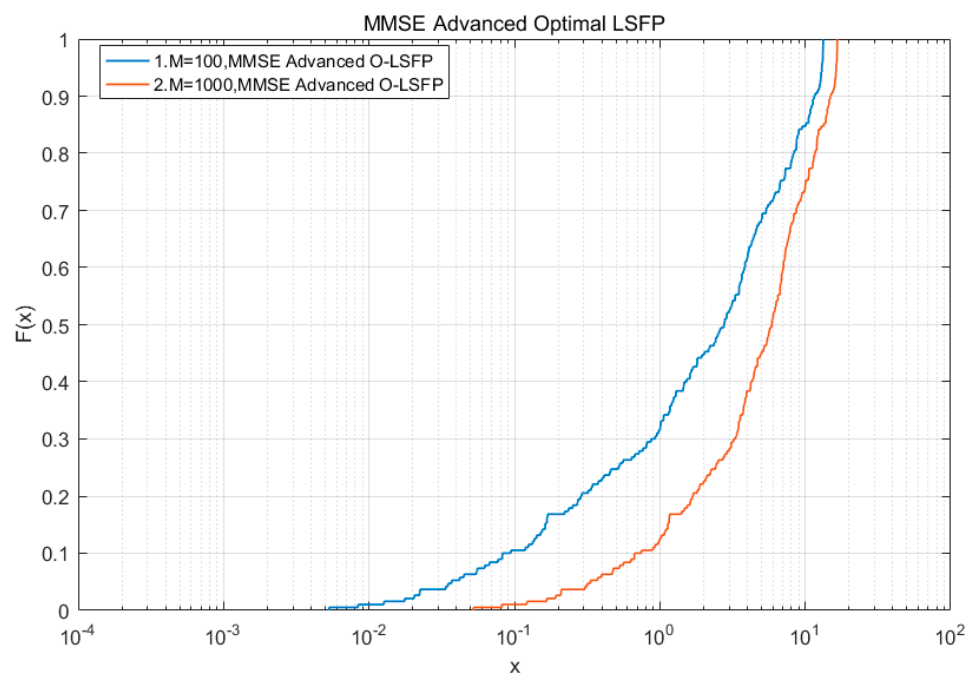

Figure 8. CDF of MMSE-A-O-LSFP channel can achieve.

\section{Conclusions}

This paper proposed and studied the anti-interference precoding algorithms that apply the channel inversion technology and use the large-scale fading factor matrix. First, channel-inversion processing was applied to the zero-forcing precoding matrix, and the advanced zero-forcing precoding algorithm is developed. Next, the advanced optimized LSFP is presented, which is based on the optimized precoding algorithm and uses the regularized pre-equalization technology. In addition, 
the MMSE pre-equalization algorithm is applied to the optimized precoding algorithm, and the advanced optimal LSFP based on the MMSE is obtained. Furthermore, the asymptotic regime is analyzed when the number of antennas of a base station approaches infinity, and it is shown that the advanced zero-forcing precoding algorithm can completely eliminate all sources of interference and additive noise. Finally, the SINR expressions for anti-interference precoding are derived and the above-improved algorithm is simulated. The simulation results show that the anti-interference precoding that use the channel inversion technology achieves the same communication transmission rate as conventional LSFP when the number of base antennas reducing. That means the improved precoding scheme have an improvement on anti-interference and improving channel transmission rate without increasing the number of base antennas in the practical cellular communication network.

Author Contributions: Conceptualization, W.L. and L.C.; methodology, L.C.; software, Y.L.; validation, W.L., Z.L. and Y.L.; formal analysis, L.Z.; investigation, Z.L.; resources, L.C.; data curation, W.L. and Z.L.; writing-original draft preparation, W.L.; writing-review and editing, L.C.; visualization, Y.L.; supervision, L.C.; project administration, L.C.; funding acquisition, L.C. All authors have read and agreed to the published version of the manuscript.

Funding: This research received no external funding.

Conflicts of Interest: The authors declare no conflict of interest.

\section{References}

1. Adhikary, A.; Ashikhmin, A.; Marzetta, T.L. Uplink Interference Reduction in Large-Scale Antenna Systems. IEEE Trans. Commun. 2017, 65, 2194-2206. [CrossRef]

2. Bjornson, E.; Sanguinetti, L.; Wymeersch, H.; Hoydis, J. Massive MIMO is a reality-What is next? Five promising research directions for antenna arrays. Digital Signal Process. 2019, 94, 3-20. [CrossRef]

3. Amiri, A.; Angjelichinoski, M.; De Carvalho, E.; Heath, R.W. Extremely Large Aperture Massive MIMO: Low Complexity Receiver Architectures. IEEE Globecom Workshops (GC Wkshps) 2018, 12, 9-13.

4. Li, L.; Ashikhmin, A.; Marzetta, T. Pilot contamination precoding for interference reduction in large scale antenna systems. Presented at the 2013 51st Annual Allerton Conference on Communication, Control, and Computing (Allerton), Monticello, IL, USA, 2-4 October 2013; pp. 226-232.

5. Ashikhmin, A.; Marzetta, T. Pilot contamination precoding in multi-cell large scale antenna systems. In Proceedings of the 2012 IEEE International Symposium on Information Theory Proceedings, Cambridge, MA, USA, 1-6 July 2012; pp. 1137-1141.

6. Björnson, E.; Hoydis, J.; Sanguinetti, L. Massive mimo has unlimited capacity. IEEE Trans. Wireless Commun. 2017, 17, 574-590. [CrossRef]

7. Ashikhmin, A.; Li, L.; Marzetta, T.L. Interference Reduction in Multi-Cell Massive MIMO Systems with Large-Scale Fading Precoding. IEEE Trans. Inf. Theory 2018, 64, 6340-6361. [CrossRef]

8. Sung, H.; Lee, S.R.; Lee, I. Generalized Channel Inversion Methods for Multiuser MIMO Systems. IEEE Trans. Commun. 2009, 57, 3489-3499. [CrossRef]

9. Yonezu, K.; Kikuma, N.; Sakakibara, K. Performance Comparison between Block Maximum SNR Algorithm and MMSE Channel Inversion Algorithm in Multiuser-MIMO Systems. In Proceedings of the IEEE 2018 International Symposium on Antennas and Propagation (ISAP), Busan, Korea, 23-26 October 2018; pp. 1-2.

10. Feryando, D.A.; Suryani, T. Performance analysis of regularized channel inversion precoding in multiuser MIMO-GFDM downlink systems. In Proceedings of the 2017 IEEE Asia Pacific Conference on Wireless and Mobile (APWiMob), Bandung, Indonesia, 28-29 November 2017; pp. 101-105.

11. Xiang, Y.; Luo, J.; Hartmann, C. Inter-cell Interference Mitigation through Flexible Resource Reuse in OFDMA based Communication Networks. European Wireless 2007, 2007, 1-7.

12. Kaltenberger, F.; Jiang, H.; Guillaud, M.; Knopp, R. Relative channel reciprocity calibration in MIMO/TDD systems. Future Netw. Mob. Summit 2010, 6, 1-10.

13. Luo, H.; Zhang, Y.; Huang, L.K.; Cosmas, J. A new reciprocity calibration method for massive MIMO systems. In Proceedings of the 2016 IEEE International Symposium on Broadband Multimedia Systems and Broadcasting (BMSB), Nara, Japan, 1-3 June 2016; pp. 1-4. 
14. Ashikhmin, A.; Marzetta, T.L. Large-scale antenna method and apparatus of wireless communication with suppression of intercell interference. U.S. Patent No 8774146, 8 July 2014.

15. Jo, H.S.; Sang, Y.J.; Xia, P.; Andrews, J.G. Heterogeneous Cellular Networks with Flexible Cell Association: A Comphrehensive Downlink SINR Analysis. IEEE Trans. Wireless Commun. 2012, 11, 3484-3495. [CrossRef]

(C) 2020 by the authors. Licensee MDPI, Basel, Switzerland. This article is an open access article distributed under the terms and conditions of the Creative Commons Attribution (CC BY) license (http://creativecommons.org/licenses/by/4.0/). 